\title{
Kinetics of polycondensation of sebacic acid with
}

\author{
glycerol
}

Supporting information

Grzegorz Matyszczak, Michał Wrzecionek, Agnieszka Gadomska-Gajadhur* and

\author{
Paweł Ruśkowski
}

Faculty of Chemistry, Warsaw University of Technology, ul. Noakowskiego 3, 00-664

Warsaw, Poland. 


\section{Prepolymer Poly(glycerol sbacate ) analysis}

Acid Number ( $\boldsymbol{A M}$ ): weigh 0.2-0.3 g of the sample, add $25 \mathrm{ml}$ of $\mathrm{MeOH}$ and 3-4 drops of thymol blue. Titrate the sample with $0.1 \mathrm{M}$ aqueous solution of $\mathrm{NaOH}$ until the colour changes from yellow to blue. Simultaneously a blank test should be assayed. The analysis was carried out in three replications. The standard error did not exceed $5 \%$.

Calculate the acid number $(A M)$ using the formula:

$$
A N=\frac{\left(V-V_{0}\right) \cdot M_{K O H} \cdot 56.1}{m} \cdot 100
$$

Where: $A N$ - acid number (mg/g sample); $V_{0}$ - volume of $0.1 \mathrm{M} \mathrm{KOH}$ used to titrate the blank test; $V$ - volume of $0.1 \mathrm{M} \mathrm{KOH}$ used to titrate the sample; $M_{\mathrm{KOH}}-\mathrm{KOH}$ titre used for titration $(0.1 \mathrm{M}) ; m$ - mass of the sample

Ester number (EN): $0.2-0.3 \mathrm{~g}$ of the sample was weighed. $15 \mathrm{~mL}$ of methanol and 20 $\mathrm{mL}$ of $0.1 \mathrm{M}$ of aqueous solution of $\mathrm{KOH}$ were added. It was heated in boiling water under a reflux condenser for 1 hour. Upon cooling the solution, the excess of added $\mathrm{KOH} 0.1 \mathrm{M}$ was titrated with hydrochloric acid in the presence of phenolphthalein. Simultaneously the blank test was conducted. The analysis was carried out in three 
replications. The standard error did not exceed $5 \%$. The ester number $(E N)$ is calculated according to the following formula:

$$
E N=\frac{\left(V_{0}-V\right) \cdot M_{H C l} \cdot 56.1}{m}-A N
$$

Where: $E N$ - ester number (mg/g sample); $A N$ - acid number; $V_{0}$ - volume $0.1 \mathrm{M} \mathrm{HCl}$ used to titrate the blank test; $V$ - volume of $0.1 \mathrm{M} \mathrm{HCl}$ used to titrate the sample; $M_{\mathrm{HCl}}$ $\mathrm{HCl}$ titre used for titration $(0.1 \mathrm{M}) ; m$ - mass of the sample

Esterification degree (ED) was calculated on the basis of the following formula:

$$
E D=\frac{E N}{E N+A N} * 100 \%
$$

Where: $E D$ - esterification degree; $A N$ - acid number; $E N$ - ester number

The conversion of sebacic acid $\mathrm{COOH}$ groups (conv $\mathrm{COOH}$ ) was calculated on the basis of the loss in mass within the post-reaction mixture as given in the following formula:

$$
\operatorname{conv} \mathrm{COOH}=\frac{\Delta m_{\text {reac }}}{\frac{2 \cdot 18}{n_{S A}}} \cdot 100 \%
$$

Where: conv $\mathrm{COOH}$ - conversion of carbonyl groups into sebacic acid; $\Delta m_{\text {reac }}$ - loss in mass of the reaction mixture; $n_{\mathrm{SA}}-$ number of moles of sebacic acid used in the reaction The analysis was carried out in three replications. The standard error did not exceed 5\%. 


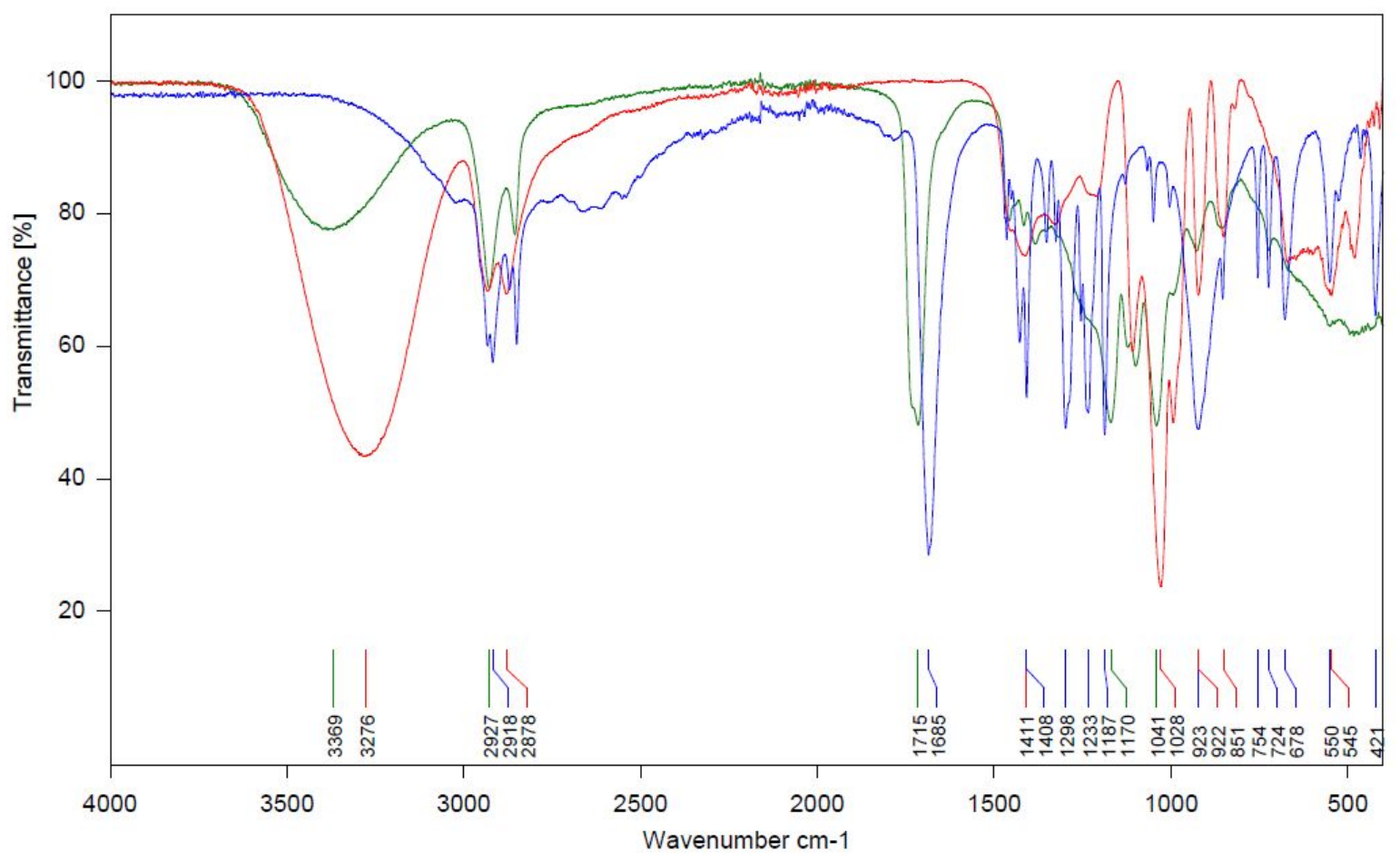

Figure S1. IR spectrums: green - obtained poly(glycerol sebacate), blue - sebacic acid,

red - glycerol 


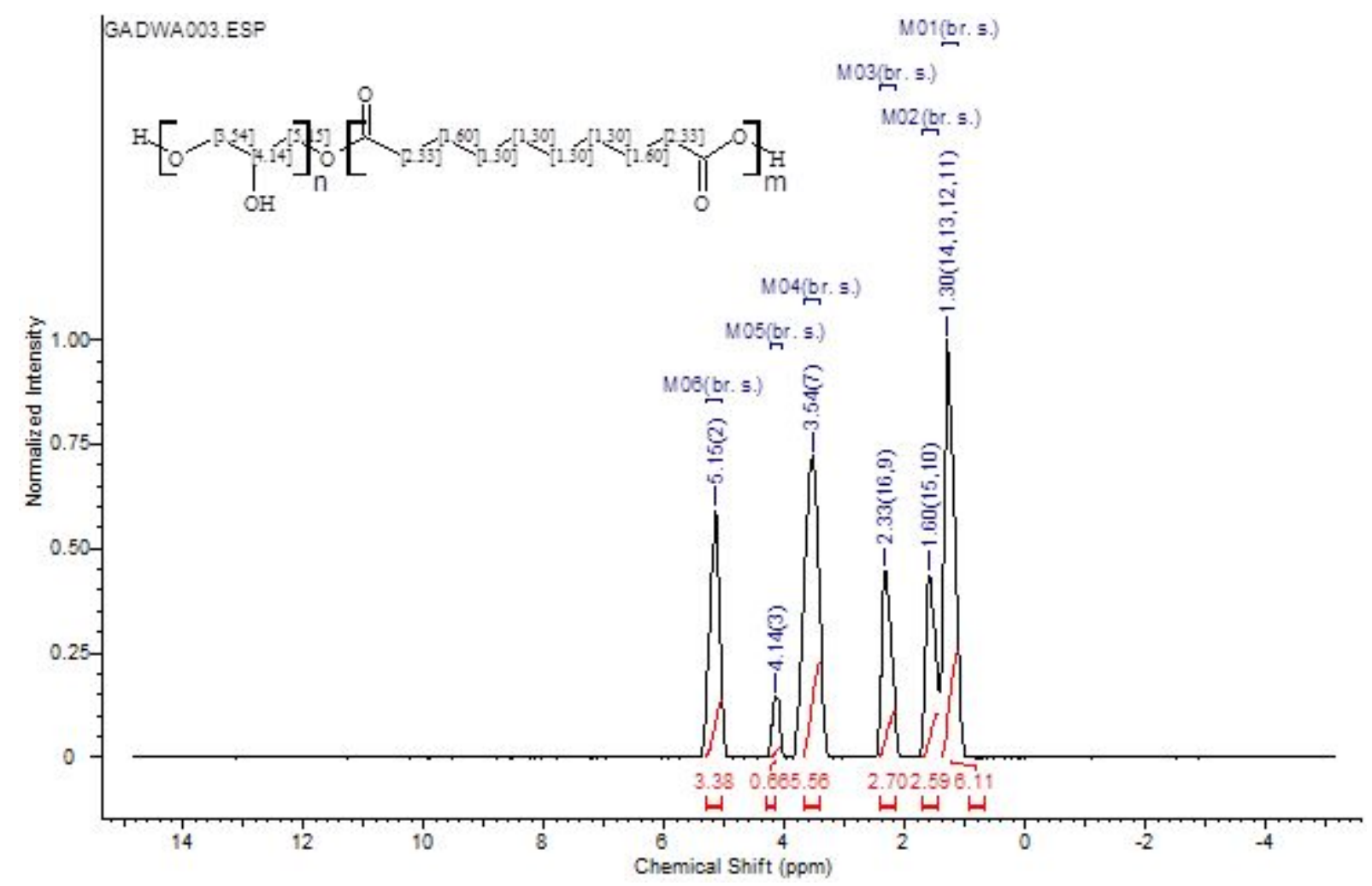

Figure S2. The polymer structure with assigned chemical shift values.

Modeling the kinetics of polycondensation

Dynamics of the reaction: due to mentioned assumptions we obtain following set of differential equations describing the dynamics of polycondensation:

$$
\begin{aligned}
& {[A]^{\prime}=-\mathrm{k}_{1}[A][B]-\mathrm{k}_{2}[A B][A]-\mathrm{k}_{5}[B A B][A]-\mathrm{k}_{6}[A B A B][A]-\mathrm{k}_{9}[B A B A B][A]} \\
& {[B]^{\prime}=-\mathrm{k}_{1}[A][B]-\mathrm{k}_{3}[A B][B]-\mathrm{k}_{4}[A B A][B]-\mathrm{k}_{7}[A B A B][B]-\mathrm{k}_{8}[A B A B A][B]} \\
& {[A B]^{\prime}=\mathrm{k}_{1}[A][B]-\mathrm{k}_{2}[A B][A]-\mathrm{k}_{3}[A B][B]} \\
& {[A B A]^{\prime}=\mathrm{k}_{2}[A B][A]-\mathrm{k}_{4}[A B A][B]} \\
& {[B A B]^{\prime}=\mathrm{k}_{3}[A B][B]-\mathrm{k}_{5}[B A B][A]} \\
& {[A B A B]^{\prime}=\mathrm{k}_{4}[A B A][B]+\mathrm{k}_{5}[B A B][A]-\mathrm{k}_{6}[A B A B][A]-\mathrm{k}_{7}[A B A B][B]} \\
& {[A B A B A]^{\prime}=\mathrm{k}_{6}[A B A B][A]-\mathrm{k}_{8}[A B A B A][B]} \\
& {[B A B A B]^{\prime}=\mathrm{k}_{7}[A B A B][B]-\mathrm{k}_{9}[B A B A B][A]} \\
& {[A B A B A B]^{\prime}=\mathrm{k}_{8}[A B A B A][B]+\mathrm{k}_{9}[B A B A B][A]}
\end{aligned}
$$


The designation $[X]^{\prime}$ means the first derivative over time. The above system of ordinary differential equations does not have an analytical solution, therefore, it should be solved numerically under 9 certain initial conditions. 
Fitting curves of absorbance over time with polynomials

\begin{tabular}{|c|c|c|c|c|c|c|c|c|c|}
\hline no & $\begin{array}{c}\boldsymbol{T}, \\
{\left[{ }^{\circ} \mathrm{C}\right]}\end{array}$ & $x^{6}$ & $x^{5}$ & $x^{4}$ & $x^{3}$ & $x^{2}$ & $x$ & $x^{0}$ & $R^{2}$ \\
\hline \multirow[b]{2}{*}{1} & 130 & $8.238 \mathrm{e}-17$ & $-1.358 \mathrm{e}-13$ & $8.964 \mathrm{e}-11$ & $-3.103 e-08$ & $6.470 \mathrm{e}-06$ & $-1.000 \mathrm{e}-03$ & $2.037 \mathrm{e}-01$ & \multirow[b]{2}{*}{0.9994} \\
\hline & $\begin{array}{l}\text { Std. } \\
\text { errors }\end{array}$ & $8.362 \mathrm{e}-18$ & $1.261 \mathrm{e}-14$ & $7.320 \mathrm{e}-12$ & $2.050 \mathrm{e}-09$ & $2.829 \mathrm{e}-07$ & $1.739 \mathrm{e}-05$ & $3.491 \mathrm{e}-04$ & \\
\hline \multirow[t]{2}{*}{2} & 130 & $\begin{array}{l}-2.150 \mathrm{e}- \\
16\end{array}$ & $2.851 \mathrm{e}-13$ & $-1.410 \mathrm{e}-10$ & $2.991 \mathrm{e}-08$ & $-1.382 \mathrm{e}-06$ & $-5.177 e-04$ & $1.614 \mathrm{e}-01$ & \multirow{2}{*}{0.9979} \\
\hline & $\begin{array}{l}\text { Std. } \\
\text { errors }\end{array}$ & $7.788 \mathrm{e}-17$ & $8.882 \mathrm{e}-14$ & $3.909 \mathrm{e}-11$ & $8.322 \mathrm{e}-09$ & $8.780 \mathrm{e}-07$ & $4.165 \mathrm{e}-05$ & $6.540 \mathrm{e}-04$ & \\
\hline \multirow[b]{2}{*}{3} & 150 & $1.708 \mathrm{e}-15$ & $-1.850 \mathrm{e}-12$ & $7.982 \mathrm{e}-10$ & $-1.775 e-07$ & $2.261 \mathrm{e}-05$ & $-1.877 \mathrm{e}-03$ & $1.721 \mathrm{e}-01$ & \multirow[b]{2}{*}{0.9989} \\
\hline & $\begin{array}{l}\text { Std. } \\
\text { errors }\end{array}$ & $1.438 \mathrm{e}-16$ & $1.441 \mathrm{e}-13$ & $5.578 \mathrm{e}-11$ & $1.046 \mathrm{e}-08$ & $9.757 \mathrm{e}-07$ & $4.110 \mathrm{e}-05$ & $5.779 \mathrm{e}-04$ & \\
\hline \multirow[b]{2}{*}{4} & 150 & $7.322 \mathrm{e}-17$ & $-1.436 \mathrm{e}-13$ & $1.130 \mathrm{e}-10$ & $-4.552 \mathrm{e}-08$ & $1.002 \mathrm{e}-05$ & $-1.267 \mathrm{e}-03$ & $1.425 \mathrm{e}-01$ & \multirow[b]{2}{*}{0.9982} \\
\hline & $\begin{array}{l}\text { Std. } \\
\text { errors }\end{array}$ & $3.882 \mathrm{e}-17$ & $4.779 \mathrm{e}-14$ & $2.268 \mathrm{e}-11$ & $5.203 \mathrm{e}-09$ & $5.906 \mathrm{e}-07$ & $3.005 \mathrm{e}-05$ & $5.042 \mathrm{e}-04$ & \\
\hline \multirow[b]{2}{*}{5} & 170 & $4.647 \mathrm{e}-15$ & $-5.069 e-12$ & $2.185 \mathrm{e}-09$ & $-4.750 \mathrm{e}-07$ & $5.564 \mathrm{e}-05$ & $-3.594 \mathrm{e}-03$ & $1.689 \mathrm{e}-01$ & \multirow[b]{2}{*}{0.9947} \\
\hline & $\begin{array}{l}\text { Std. } \\
\text { errors }\end{array}$ & $3.759 \mathrm{e}-16$ & $3.643 \mathrm{e}-13$ & $1.364 \mathrm{e}-10$ & $2.476 \mathrm{e}-08$ & $2.236 \mathrm{e}-06$ & $9.135 \mathrm{e}-05$ & $1.248 \mathrm{e}-03$ & \\
\hline \multirow[b]{2}{*}{6} & 170 & $2.487 \mathrm{e}-15$ & $-3.118 \mathrm{e}-12$ & $1.538 \mathrm{e}-09$ & $-3.794 e-07$ & $4.978 \mathrm{e}-05$ & $-3.575 e-03$ & $1.811 \mathrm{e}-01$ & \multirow[b]{2}{*}{0.9932} \\
\hline & $\begin{array}{l}\text { Std. } \\
\text { errors }\end{array}$ & $1.721 \mathrm{e}-16$ & $1.958 \mathrm{e}-13$ & $8.592 \mathrm{e}-11$ & $1.825 \mathrm{e}-08$ & $1.920 \mathrm{e}-06$ & $9.086 \mathrm{e}-05$ & $1.424 \mathrm{e}-03$ & \\
\hline
\end{tabular}

Table S1. The correlation coefficients of fitted sixth degree polynomials and their standard errors at three tested temperatures. 